\title{
Ekkwill Zebrafish
}

National Cancer Institute

\section{Source}

National Cancer Institute. Ekkwill Zebrafish. NCI Thesaurus. Code C79971.

A wild-type zebrafish line, the stock of which was obtained from Ekkwill Breeders (Florida). 